and designing for construction ergonomics skills, resonates with the findings of other research. There is a need for construction ergonomics to be embedded in tertiary built environment programmes, ergonomics continuing professional development (CPD), a construction industry ergonomics standard, and ergonomics practice notes.

\section{SECONDARY PREVENTION OF LOW BACK PAIN IN THE OCCUPATIONAL HEALTH: EFFECTIVENESS AND COST- EFFECTIVENESS OF AN EARLY MANAGEMENT PROGRAM}

\begin{abstract}
1,2,3 J Rantonen*, 4,5,6 J Karppinen, ${ }^{7} \mathrm{~A}$ Vehtari, ${ }^{8,9,10} \mathrm{~S}$ Taimela. ${ }^{1}$ Department of Occupational Medicine, South Karelia Social and Health Care District, Lappeenranta, Finland; ${ }^{2}$ Lappeenranta University of Technology, Lappeenranta, Finland; ${ }^{3}$ University of Helsinki, Faculty of Medicine, Helsinki, Finland; ${ }^{4}$ Medical Research Centre Oulu, University of Oulu, Finland; ${ }^{5}$ Oulu University Hospital, Oulu, Finland; ${ }^{6}$ Finnish Institute of Occupational Health, Helsinki, Finland; ${ }^{7}$ Helsinki Institute for Information Technology HIIT, Department of Computer Science, Aalto University, Espoo, Finland; ${ }^{8}$ Evalua International, Espoo, Finland; ${ }^{9}$ Department of Orthopaedics and Traumatology, Helsinki University Hospital, Finland; ${ }^{10}$ University of Helsinki, Helsinki, Finland
\end{abstract}

\subsection{6/oemed-2018-ICOHabstracts.790}

Introduction Low back pain (LBP) is one of the leading causes of disability all over the world. We performed a secondary prevention program of LBP among employees that reported mild or moderate level low back symptoms in a large forestry industry complex.

Methods First, respondents of an employee survey $(n=2480$; response rate $71 \%$ ) were eligible into this study, if they fulfilled pre-defined low back (LB) specific risk assessment criteria. Secondly, eligible employees $(n=505,66 \%$ males, 45 y) were divided into two sub-cohorts, 'Mild' and 'Moderate' LBP, according to recent $\mathrm{LB}$ pain intensity. Sub-cohort Mild $(n=181,47$ refused) was randomised into two intervention arms, both receiving back book information and the other arm also additional face-to-face patient information. Subcohort Moderate ( $n=126,17$ refused) was randomised into three groups, receiving either one of two active exercise interventions or $\mathrm{LB}$ specific advice from their occupational health $(\mathrm{OH})$ physician. All intervention arms in Mild and Moderate were controlled by their respective natural course (NC) of LBP groups $(n=83$ and $n=50$, respectively). Primary outcomes were disability (Roland-Morris Disability Questionnaire (0-18) and Oswestry Disability sum index, 0-50), LB pain (Visual Analogue Scale, $0-100 \mathrm{~mm}$ ) and total sickness absence days (SA).

Results Mild: Compared to NC, pain, disability and SA decreased after both interventions and back book information alone was also cost-effective. Moderate: Compared to NC, pain and disability decreased after both active interventions but SA did not. OH physician's advice was not effective. Interventions in Moderate were not cost-effective in two years.

Discussion Simple patient information was effective and also cost-effective in mild LBP. Active LB specific interventions were effective but not cost-effective after two years in moderate LBP. OH physician's advice was not effective. Population based LB specific risk assessment seems feasible. In general, proactive management of LBP is recommendable in the $\mathrm{OH}$ setting.

\section{WORK-BREAK SCHEDULES FOR PREVENTING MUSCULOSKELETAL DISORDERS IN WORKERS - A COCHRANE REVIEW}

${ }^{1}$ Tessy Luger*, ${ }^{2}$ Chris G Maher, ${ }^{1}$ Monika A Rieger, ${ }^{1}$ Benjamin Steinhilber. ${ }^{1}$ Institute of Occupational and Social Medicine and Health Services Research, Tuebingen, Germany; ${ }^{2}$ Sydney School of Public Health, The University of Sydney, Sydney, Australia

\subsection{6/oemed-2018-ICOHabstracts.791}

Introduction Repetitive and monotonous work, especially manual work, is very common in modern industrial operations, resulting in an increased risk of musculoskeletal disorders. It is therefore important to find an appropriate intervention counteracting or preventing the repetitive and monotonous character of work tasks, for example by work-breaks. This review aims to assess the effectiveness of work-breaks (compared to no work-breaks or regular work-break schedules) for preventing work-related musculoskeletal disorders in workers. A work-break can be defined as any scheduled work-interruption that is not related to work, which includes the following characteristics: frequency (amount, timing), duration, or type (e.g. active or passive).

Methods We will search the literature (e.g., CENTRAL, PubMed) for randomised, quasi-randomised, and cluster-randomised studies without language restrictions. We will include trials that have enrolled adult workers without musculoskeletal symptoms and that have assessed one or more of the following work-break interventions: changes in break duration, frequency, timing, or type. Two review authors will independently consider retrieved records for eligibility and extract the data.

Result The extracted data will be summarised and two review authors will independently assess the risk of bias for each study regarding random sequence allocation, allocation concealment, blinding of participants and personnel, blinding of outcome assessment, incomplete outcome data, and selective outcome reporting (criteria as outlined in the Cochrane Handbook). The meta-analysis will initially be performed including all studies. Thereafter a sensitivity analysis confined to trials at low risk of bias will be conducted. The heterogeneity of the results of included studies will be assessed by visual inspection of the forest plots and consideration of trial characteristics, e. g. work-break characteristics.

Discussion The results of this Cochrane Review will provide insights into the effectiveness of work-break interventions and provide direction for optimising current prevention approaches and help prioritise future fields of research.

\section{REDUCTION OF LEG SWELLING BY COMPRESSION STOCKINGS WITH DIFFERENT COMPRESSION INTENSITIES DURING A TWO HOUR STANDING EXPOSURE - A PILOT STUDY}

Benjamin Steinhilber*, Angela Enghofer, Robert Seibt, Monika A Rieger. Institute of Occupational and Social Medicine and Health Services Research, Tuebingen, Germany

\subsection{6/oemed-2018-ICOHabstracts.792}

Introduction Lower leg swelling is considered to be a risk factor of venous disorders among workers exposed to prolonged standing. Compression stockings might be effective in reducing lower leg swelling; however, little is known about the effect of different compression intensities. The aim of this study was 
to investigate the influence of two different compression intensities throughout a 2 hour standing exposure on lower leg swelling, complaints, and wearing comfort.

Methods 40 healthy subjects participated in this randomised cross-over experiment with three 2 hour standing exposures that were tested on separate days. In condition A, subjects did not wear compression stockings; in condition B and C, subjects wore compression stockings medical class I (18$21 \mathrm{mmHg})$ and class II $(23-32 \mathrm{mmHg})$, respectively. Lower leg swelling was quantified by measures of lower leg volume (water plethysmography) and bioelectrical impedance before and after each standing exposure. Level of discomfort was assessed every $30 \mathrm{~min}$ (11-point Likert-Scale) and wearing comfort was measured at the end of the exposure using a custom-made standardised questionnaire. The study was approved by the ethics committee of the University of Tuebingen.

Result Preliminary results $(n=19)$ indicated that wearing compression stockings reduced lower leg swelling compared to wearing no stockings, but there was no difference in leg swelling between the two compression classes. Levels of discomfort were rather low and did not significantly differ across conditions. Higher levels of wearing comfort were found for the class I compared to the class II stockings.

Discussion These preliminary results suggest that in healthy subjects the lower compression intensity might be as effective as the higher intensity in reducing lower leg swelling. This is an important finding since compliance of wearing compression stockings increases with lower compression intensities, which is supported by the increased wearing comfort for the lower compression class in this study.

\section{POLITICS IN OCCUPATIONAL HEALTH: EXAMPLE OF AN EVIDENCE-BASED DRIVEN PROPOSAL FOR IMPROVING UPPER-EXTREMITY MUSCULOSKELETAL DISORDER COMPENSATION IN FRANCE}

1,2Alexis Descatha*. 'AP-HP, EMS (Samu92), Occupational Health Unit, University hospital of West suburb of Paris, Poincare site, F92380 Garches, France; ${ }^{2}$ Univ Versailles St-Quentin, F-78035, Versailles, France; Inserm, UMS 011 UMR1168, F-94807, Villejuif, France

\subsection{6/oemed-2018-ICOHabstracts.793}

Introduction In a context of heterogeneous practices, the French government set up a committee and this committe have mandated a technical working group for improving compensation for upper-extremity musculoskeletal disorders.

Methods After a state of the art based on international recommendations and French data to take into account the particular nature of compensation in France, a technical working group followed a Delphi-like process for suggesting a method of evaluation. A different group evaluated the work at the end with a validation process.

Result A systematic review has been performed, supplemented with opinions of International experts solicited individually. Analyses of available data were carried out on actual compensation and on a French cohort, as well as an inventory of the various French compensation methods. From this state of the art, the technical working group proposed a first version which was discussed in a workshop, allowing after two rounds a draft that a majority agreed upon $(>70 \%$ of total agreement). An independent group read and evaluated the work, with over 90\% total agreement, and allowed minor comments before the final report.

Discussion In one year, this project allowed the development of an evidence-based approach aimed at improving compensation for upper-extremity musculoskeletal disorders. An appropriation phase is necessary involving by end-users (i.e. insurance practitioners, with development of practical tools, clinical vignettes), and an economical evaluation.

\section{WORK EXPOSURES AND THEIR RELATIONSHIP TO THE DEVELOPMENT OF OSTEOARTHRITIS: A SYSTEMATIC REVIEW}

${ }^{1}$ Emma Irvin*, 'Monique Gignac, ${ }^{1}$ Kimberley Cullen, 'Dwayne Van Eerd, ${ }^{2}$ Catherine Backman, ${ }^{3}$ Dorcas Beaton, ${ }^{1}$ Siobhan Cardoso, ${ }^{1}$ Quenby Mahood, ${ }^{2}$ Christopher McLeod. ${ }^{1}$ Institute for Work and Health, Toronto, Canada; ${ }^{2}$ University of British Columbia, Vancouver, Canada; ${ }^{3}$ University of Toronto, Toronto, Canada

\subsection{6/oemed-2018-ICOHabstracts.794}

Introduction Osteoarthritis (OA) ranks among the top ten causes of disability worldwide. Of increasing interest are occupational factors that contribute to the development of $\mathrm{OA}$ or aggravate its trajectory. This systematic review synthesised evidence for the relationship between work exposures and the development of OA to inform work disability prevention messaging and decision making and to identify future knowledge needs.

Methods We implemented the systematic review process developed by the Institute for Work and Health and an adapted best evidence synthesis. Four electronic databases were searched from inception until May 2015. Articles that described the impact of work on OA were included in the review and examined knees, hips, spine, wrist, hands, or fingers, shoulder, ankle, foot, or toes, neck, elbow.

Result The search yielded 3379 non-duplicate references with 67 studies meeting our inclusion criteria. Study designs included cross-sectional surveys $(n=22)$, case-control studies $(n=22)$, prospective cohorts $(n=14)$, and retrospective cohorts $(n=9) .96 \%$ of studies measured OA using reliable and valid measures. Measurement of work exposures was more variable with $33 \% \quad(n=28)$ of studies not well described and $18 \%$ $(n=12)$ using instruments with questionable or unknown reliability and validity. There was strong or moderate evidence of an increased risk for developing OA in hips or knees for several occupational tasks (e.g., lifting/load bearing activities, full body vibration, kneeling/bending/squatting) and for not having an increased risk of OA for walking, sitting, driving, climbing. Dose response data were highly variable and could not be synthesised for recommendations.

Discussion This systematic review highlights that strong evidence exists for occupational risks and the development of $\mathrm{OA}$ in some joints, like knees and hips. However, data on complex job tasks (e.g., bending and lifting simultaneously) and dose response information are lacking. This is essential information going forward for prevention and early intervention efforts. 